\title{
Spectrum Extraction for 2-D Fiber Spectrum Images Based on 2-D Gaussian Model
}

\author{
Zhangqin Zhu ${ }^{\mathrm{A}}$, Jia Zhu ${ }^{\mathrm{A}}$, Sheng Wang ${ }^{\mathrm{A}}$, Shenghong Cao ${ }^{\mathrm{A}}$, \\ Guangzhao Bao ${ }^{\mathrm{A}}$, and Zhongfu Ye $\mathrm{A}^{\mathrm{B}}$ \\ ${ }^{A}$ Institute of Statistical Signal Processing, University of Science and Technology \\ of China, Hefei 230027, China \\ ${ }^{\mathrm{B}}$ Corresponding author. Email: yezf@ustc.edu.cn
}

\begin{abstract}
A novel spectrum-extraction method based on a 2-D Gaussian model is proposed in this paper. First, the flat images are employed to fit the model parameters in the spatial orientation and the calibration lamp images are used to fit the model parameters in the wavelength orientation. Then normalized 2-D models are obtained by combining the parameters of the two orientations. The flux-extraction algorithm is based on least-square theory and the 2-D model. Through experiments, the extracted spectra by our method have a stronger ability to reduce noise than the 1-D spectrum extraction method.
\end{abstract}

Additional keywords: line: profiles — methods: data analysis — techniques: spectroscopic

Received 2010 December 29, accepted 2011 September 15, published online 2011 October 26

\section{Introduction}

Spectrum extraction is one of the most important steps for the processing of 2-D fiber spectrum images. The precision of flux extraction plays an important role in the subsequent processing. For now, there are two main kinds of extraction methods: one is the aperture extraction method and the other is the profile-fitting method.

The aperture extraction method (de Boer \& Snijders 1981) is a simple method, and it works rapidly. This method counts the flux within a certain aperture around the fiber profile center in the spatial orientation, so the noise and cross-talk of adjacent fibers are all extracted as the fiber flux. Existing aperture extraction methods (Horne 1986; Robertson 1986; Marsh 1989) employ different weightings of different pixels to restrain the effects of noise and cross-talk. Other developments, such as creating an instrumental profile database (Becker 2001; Roth et al. 2005) have been proposed for each CCD to improve the accuracy of spectrum extraction, which would require an accurate determination of the profiles (Sanchez 2006).

The profile-fitting method (Piskunov \& Valenti 2002; Blondin et al. 2005) is another spectrum-extraction method, which uses a 1-D function (usually a Gaussian function) to fit the profile of fiber flux in the spatial orientation. The effectiveness of this method is dependant on the signal-to-noise ratio $(\mathrm{S} / \mathrm{N})$ of the data (Sanchez 2006). This method is widely used to extract spectra for fiber images such as the Sloan Digital Sky Survey (SDSS) and the Large Sky Area Multi-Object Fiber Spectroscopy Telescope (LAMOST). An improved method named Gaussian suppression combines the aperture method and the Gaussian fitting method (Sanchez 2006), extracting the flux in the spatial orientation. The radial basic function method (Qin et al. 2009) uses two Gaussian functions to fit profiles for LAMOST. All these profilefitting methods fit the fiber profile only in the spatial orientation, but in fact, when the flux is projected onto the $\mathrm{CCD}$, theenergy diffuses to the adjacent pixels in the form of a 2-D point spread function (PSF), not only in the spatial direction. If the spectra can be extracted by 2-D PSF, the accuracy of the extraction should be improved. An algorithmic framework (Bolton \& Schlegel 2010) was proposed based on accurate 2-D forward modeling of the raw pixel data, in which the algorithm is correct for arbitrarily complicated 2-D PSFs. An optimal extraction methodology (Sharp \& Birchall 2010) was presented for multi-fiber spectroscopy. The method can achieve high accuracy and minimize the impact of fiber-to-fiber crosscontamination.

In this paper, a novel spectrum extraction method based on a normalized 2-D Gaussian model is proposed. The variance of the model in the spatial and wavelength orientation is figured out from the flat image and calibration lamp image, respectively. Then the two parameters are combined to count the normalized 2-D PSF model. For the observed image, sufficient sampling points are adopted to get the extracted flux based on least-squares theory and the normalized 2-D model. Experiments in this paper demonstrate the effectiveness of our novel method.

The parameters obtaining to the 2-D Gaussian model and the spectrum extraction method are described in Section 2. In Section 3, experiments and results are 
given. A summary is presented in the last section of this article.

\section{Spectrum Extraction Method}

\subsection{The Parameters of the 2-D Gaussian Mode}

For now, the profile-fitting method only fits the fiber profile in the spatial orientation, and the extracting model is a Gaussian function.

$$
\begin{aligned}
f(x, y) & =\frac{A}{\sqrt{2 \pi} \sigma} e^{-\frac{\left(x-x_{c e n}\right)^{2}}{2 \sigma^{2}}} \\
\sigma & =\frac{F W H M}{2 \sqrt{\ln 4}}
\end{aligned}
$$

where $x$ denotes the pixel of the spatial orientation and $y$ is the wavelength orientation. FWHM denotes the full width at half maximum of the Gaussian function. $\sigma^{2}$ is the variance of the Gaussian function.

Our proposed method employs a normalized 2-D Gaussian function to extract the fiber flux. The model can be described as,

$$
\begin{aligned}
f(x, y) & =\frac{A}{2 \pi \sigma_{1} \sigma_{2}} e^{-\frac{\left(x-x_{c e n}\right)^{2}}{2 \sigma_{1}^{2}}-\frac{\left(y-y_{c e n}\right)^{2}}{2 \sigma_{2}^{2}}} \\
\sigma_{1} & =\frac{F W H M_{1}}{2 \sqrt{\ln 4}} \\
\sigma_{2} & =\frac{F W H M_{2}}{2 \sqrt{\ln 4}}
\end{aligned}
$$

where $x, y$ denote the pixel of the spatial and wavelength orientation, respectively. $\mathrm{FWHM}_{1}, \mathrm{FWHM}_{2}$ denote the full width at half maximum of the Gauss function in the spatial and wavelength orientations, respectively. $\sigma_{1}^{2}, \sigma_{2}^{2}$ denote the variances of the Gaussian function in the spatial and wavelength orientations, respectively.

The FWHM may be different in the spatial and wavelength directions of the 2-D model, but it may also be the same in both directions. The model can work well in either case. $\sigma_{1}$ can be figured out based on the flat image by least-squares theory. For each fiber, the centers are first obtained by the gravity method (Zhang et al. 2001). Along the wavelength orientation, several points around each center of the fiber are adopted to figure out the $\sigma_{1}$ as follows:

$$
f\left(x_{i}, y_{j}\right) \approx \frac{A_{j}}{\sqrt{2 \pi} \sigma_{j}} e^{-\frac{\left(x_{i}-x_{c e n}\right)^{2}}{2 \sigma_{j}^{2}}}
$$

where $x_{i}$ is the sampling point in the spatial orientation at the $j$ th center of the fiber.

In this step, the FWHM is the object of our interest. The coefficient of the exponential function is disregarded and its value does not affect the FWHM. The above formula can be described as,

$$
\begin{aligned}
\ln f\left(x_{i}, y_{j}\right) & \approx B+\ln e^{-A\left(x_{i}-x_{c e n}\right)^{2}} \\
B & =\ln \frac{A_{j}}{\sqrt{2 \pi} \sigma_{j}} \\
A & =\frac{1}{2 \sigma_{j}^{2}}
\end{aligned}
$$

Least-squares theory is employed to estimate the parameters of the above formula.

$$
\sum_{i=1}^{K}\left[\ln f\left(x_{i}, y_{j}\right)-\left(B+\ln e^{-A\left(x_{i}-x_{c e n}\right)^{2}}\right)\right]^{2} \rightarrow \min
$$

where $K$ is the number of sampling points of the spatial orientation at the $j$ th center of the fiber.

The value of $\sigma_{1}$ may be different for different centers along the wavelength orientation for the fiber. To satisfy the above formula,

$$
\begin{aligned}
& \frac{\partial \sum_{i=1}^{K}\left[\ln f\left(x_{i}, y_{j}\right)-\left(B+\ln e^{-A\left(x_{i}-x_{c e n}\right)^{2}}\right)\right]^{2}}{\partial A}=0 \\
& \frac{\partial \sum_{i=1}^{K}\left[\ln f\left(x_{i}, y_{j}\right)-\left(B+\ln e^{-A\left(x_{i}-x_{c e n}\right)^{2}}\right)\right]^{2}}{\partial B}=0 .
\end{aligned}
$$

Then, $A$ and $B$ can be obtained according to

$$
\begin{aligned}
& {\left[\begin{array}{cc}
K & \sum_{i=1}^{K}\left(x_{i}-x_{c e n}\right)^{2} \\
\sum_{i=1}^{K}\left(x_{i}-x_{c e n}\right)^{2} & \sum_{i=1}^{K}\left(x_{i}-x_{c e n}\right)^{4}
\end{array}\right]\left[\begin{array}{l}
B \\
A
\end{array}\right]} \\
& =\left[\begin{array}{c}
\sum_{i=1}^{K} \ln \left(f\left(x_{i}, y_{j}\right)\right) \\
\sum_{i=1}^{K}\left[\ln \left(f\left(x_{i}, y_{j}\right)\right) \times\left(x_{i}-x_{c e n}\right)^{2}\right]
\end{array}\right] .
\end{aligned}
$$

And the $\sigma_{j}$ can be calculated as

$$
\sigma_{j}=\frac{1}{\sqrt{2 A}}
$$

$\sigma_{2}$ can be obtained from the calibration lamp image based on least-squares theory. The emission lines need to be chosen to avoid blend emissions which will increase the FWHM in the wavelength direction. For each fiber, after calibrating the centers along the wavelength orientation, the flux of the centers is extracted to form a 1-D signal. Due to the fluxes of adjacent wavelength pixels mixing, the emission lines are chosen to fit the FWHM of the wavelength of the 2-D model. For the same fiber, emission lines are always much stronger than the continuous spectrum, so the profile effect of these emission lines by their adjacent continuous spectrum is not obvious. To most weaken the effect of continuous spectrum, the extracting signal will subtract its values after median filtering.

From Figure 1, we can see that, in the extracted signal after subtracting the result of its own median filtering, the 


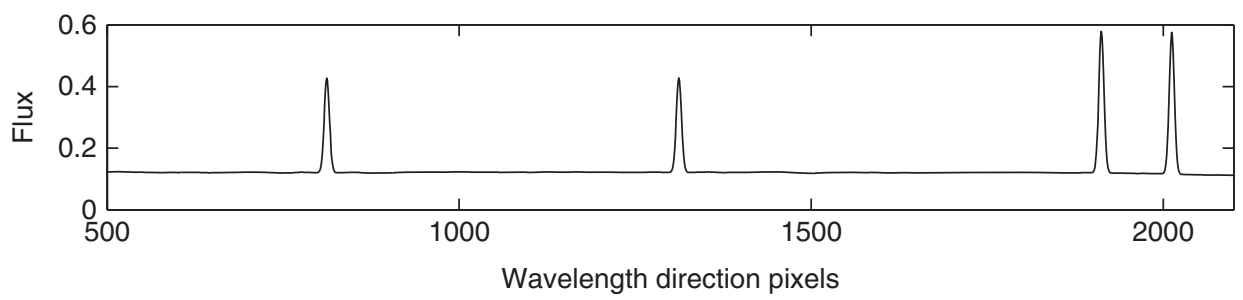

(a)

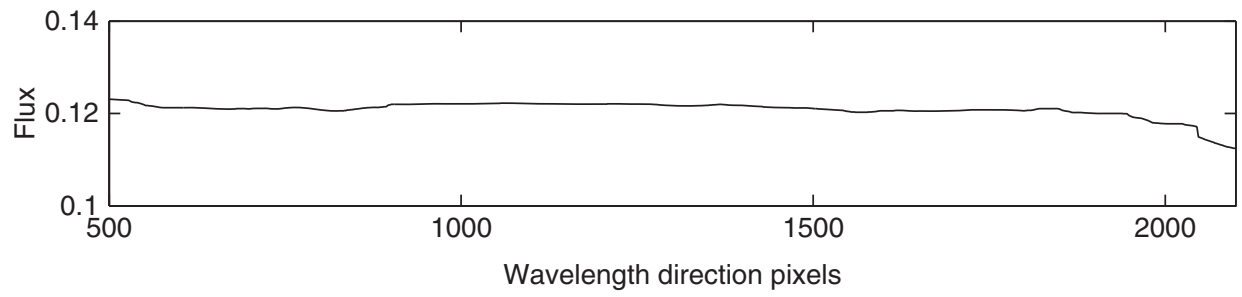

(b)

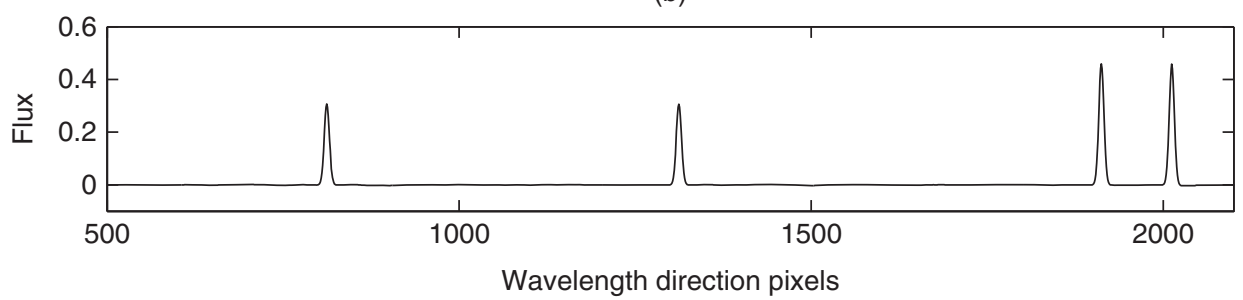

(c)

Figure 1 The signal extracted from the centers of the fiber along the wavelength. (a) The extracting signal. (b) The median filtering of (a). (c) The signal after (b) is subtracted from (a).

Table 1. The $\sigma_{2}$ estimated by this method in wavelength orientation

\begin{tabular}{lllll}
\hline$\sigma_{2}^{0}$ & 4.2466 & 4.2466 & 4.2466 & 4.2466 \\
$\sigma_{2}^{1}$ & 4.2498 & 4.2479 & 4.2468 & 4.2351 \\
$\sigma_{2}^{2}$ & 5.4669 & 5.4758 & 5.0902 & 5.3764 \\
\hline
\end{tabular}

$\sigma_{2}^{0}$ denotes the true value.

$\sigma_{2}^{1}$ is the estimated value after median filtering.

$\sigma_{2}^{2}$ is the estimated value without filtering.

continuous spectrum is almost at 0 , and the peak heights of emission lines are also reduced.

The fiber is simulated without noise, in which the FWHM of the wavelength orientation is 10 pixels. From Table 1 , we can see that after median filtering, $\sigma_{2}^{1}$ is closer to the true value. $\sigma_{2}$ is considered to be the same for the same fiber.

The parameters $\sigma_{1}$ and $\sigma_{2}$ are obtained already, and according the Equation 2, the normalized 2-D Gaussian model can be calculated.

\subsection{Spectrum Extraction}

The normalized 2-D Gaussian model has been set up. To simplify the algorithm, the cross-talk is considered to be ignored for the middle points of the fiber profiles. The more complex situation will be discussed later.

For a fiber, $M$ denotes the pixel numbers in wavelength orientation. $N$ denotes the sampling points from the observed fiber, and these points should be close to the centers of the fiber to avoid the cross-talk of adjacent fibers. As the value of $N$ becomes larger, the information of profiles is more integrated, but the effect of noise and cross-talk is heavier. The choice of $N$ is dependant on the signal-to-noise and the degree of cross-talk of the observed images. $N$ must be larger than $M$ to employ leastsquares theory.

For each sampling point, $y_{i},(i=1, \ldots, N)$ denotes the observed value. $G_{j i},(j=1, \ldots, M ; i=1, \ldots, N)$ denotes the 2-D Gaussian model of the $j$ th center along the wavelength orientation of the fiber projecting onto the $i$ th sampling point. $x_{j}(j=1, \ldots, M)$ denotes the original value of the $j$ th center along the wavelength orientation before diffusing in the form of the 2-D PSF, and the $x_{j}$ is the flux extracted. Because of the noise and other effects, the error information is addressed to improve the accuracy of spectrum extraction. It is assumed that Poisson statistics describe the observed count rate, in combination with the measured CCD gain and read-noise parameters.

Each sampling point can be described by the formula

$$
\frac{\sum_{j=1}^{M} x_{j} G_{j i}}{\sigma_{i}}=\frac{y_{i}}{\sigma_{i}}
$$

where

$$
\sigma_{i}=\sqrt{\frac{y_{i} \times \text { gain }+N_{r d}^{2}}{\text { gain }}},
$$

gain denotes measured CCD gain, and $N_{\text {rd }}$ denotes read noise. 
The above formula can be written in the form of a matrix.

$$
\mathrm{GX}=\mathrm{Y}
$$

where

$$
\begin{gathered}
\mathrm{G}=\left[\begin{array}{cccc}
G_{11} / \sigma_{1} & G_{21} / \sigma_{1} & \cdots & G_{M 1} / \sigma_{1} \\
G_{12} / \sigma_{2} & G_{22} / \sigma_{2} & \cdots & G_{M 2} / \sigma_{2} \\
\vdots & \vdots & \ddots & \vdots \\
G_{1 N} / \sigma_{N} & G_{2 N} / \sigma_{N} & \cdots & G_{M N} / \sigma_{N}
\end{array}\right] \\
\mathrm{X}=\left[\begin{array}{c}
\mathrm{x}_{1} \\
\mathrm{x}_{2} \\
\vdots \\
\mathrm{x}_{\mathrm{M}}
\end{array}\right], \mathrm{Y}=\left[\begin{array}{c}
\mathrm{y}_{1} / \sigma_{1} \\
\mathrm{y}_{2} / \sigma_{2} \\
\vdots \\
\mathrm{y}_{\mathrm{N}} / \sigma_{\mathrm{N}}
\end{array}\right]
\end{gathered}
$$

where $\mathbf{G}$ has the dimensions $N \times M$, and $\mathbf{X}, \mathbf{Y}$ are vectors of dimensions $M \times 1$ and $N \times 1$, respectively.

The propagation of error information is important for all observational science. In our method, the propagation of error information can be calculated as follows.

For a sampling point, the value is considered to be the mixing of many 2-D model with different contributions.

$$
\sum_{j=1}^{M} x_{j} G_{j i}=y_{i}
$$

For each 2D model, its fractional contribution is

$$
F_{j i}=\frac{x_{j} G_{j i}}{y_{i}} .
$$

An estimate of the total variance in the extracted intensity of $x_{j}$ is then given by

$$
\operatorname{var}\left(x_{j}\right)=\sum_{i} F_{j i}^{2} \times \sigma_{i}^{2}
$$

Equation 10 is over-determined, and the least-squares solution is our objective to satisfy Equation 10.

$$
\|\mathrm{GX}-\mathrm{Y}\|_{2} \rightarrow \min ,
$$

where $\|\cdot\|_{2}$ denotes the least-squares limit (second-order norm limit). The solution of Equation 11 under the least 2norm limit is introduced as follows. G can be decomposed by singular value decomposition (SVD) to

$$
\begin{aligned}
& \mathrm{G}=\mathrm{U} \Sigma \mathrm{V}^{\mathrm{H}} \\
& \Sigma=\left[\begin{array}{c}
\Sigma_{1} \\
0
\end{array}\right], \Sigma_{1}=\operatorname{diag}\left(\delta_{1}, \cdots, \delta_{M}\right)
\end{aligned}
$$

where $\mathbf{U}$ is the orthogonal matrix with $N \times N$ while $\mathbf{V}$ is the orthogonal matrix with $M \times M$, and $\Sigma$ is the matrix with $N \times M$. $\operatorname{diag}\left(\delta_{1}, \ldots, \delta_{M}\right)$ denotes a square matrix with $M \times M$, and its diagonal elements are in the sequence $\delta_{1}>0, \ldots, \delta_{M}>0$ while other elements are $0 . \mathbf{V}^{H}$ denotes the conjugated and transposed matrix of $\mathbf{V}$.

Equation 11 can be described as,

$$
\begin{gathered}
\|\mathrm{GX}-\mathrm{Y}\|_{2}=\left\|\mathrm{U} \Sigma \mathrm{V}^{H} \mathrm{X}-\mathrm{Y}\right\|_{2} \\
=\left\|\Sigma \mathrm{V}^{H} \mathrm{X}-\mathrm{U}^{H} \mathrm{Y}\right\|_{2}=\|\Sigma \mathrm{x}-\mathrm{c}\|_{2} \\
\mathrm{x}=\mathrm{V}^{H} \mathrm{X}, \mathrm{c}=\mathrm{U}^{H} \mathrm{Y}
\end{gathered}
$$

The solution of $\mathbf{x}$ can be obtained from Equation 14,

$$
x=\left[c_{1} / \delta_{1}, c_{2} / \delta_{2}, \cdots, c_{M} / \delta_{M}\right]
$$

where $\mathbf{x}$ is a vector with $M$ elements.

The solution of $\mathbf{X}$ can be obtained from Equation 15,

$$
\mathrm{X}=\mathrm{Vx}
$$

The solution of $\mathbf{X}$ is the result of flux extraction.

The speed of the algorithm is a factor of concern. For one fiber with 2500 pixels in the wavelength direction and 12500 sampling points, the time of spectrum extraction is about 30 seconds to 40 seconds on a computer running Windows XP system with an AMD dual-core processor $5000+$ and 2 GB RAM.

\section{Experiments and Discussion}

In this section, $M$ is the number of pixels of a fiber in the wavelength orientation, and its value is $2500 . N$ is the number of sampling points, and its value is 12500 .

\subsection{Flux Extraction without Noise}

In this part, one standard spectrum is adopted from one standard data measurement. The spectrum diffuses to adjacent pixels along the fiber centers in the form of a 2-D Gaussian PSF. It is assumed that cross-talk and noise are ignored. In this subsection, flux extraction only in the spatial orientation and the aperture extraction method are chosen to be the contrast methods. The original spectrum and the extraction flux are shown in Figure 2 as the normalized form.

In Figure 3, the errors of extracted spectra are shown, and from the figure, we can see that the three methods have similar errors.

\subsection{Flux Extraction with Noise}

In this part noise is added into the 2-D spectra. The noise is composed of two parts, Poisson noise and Gaussian random noise. The extracted spectra by our method, the single-Gaussian method and the aperture-extraction method are shown in Figure 4.

From Figure 4, the single-Gaussian method and the aperture-extraction method are significantly affected by noise, while the results of our method are polluted by noise only slightly.

The SNR of the observed 2-D fiber can be calculated as follows. 

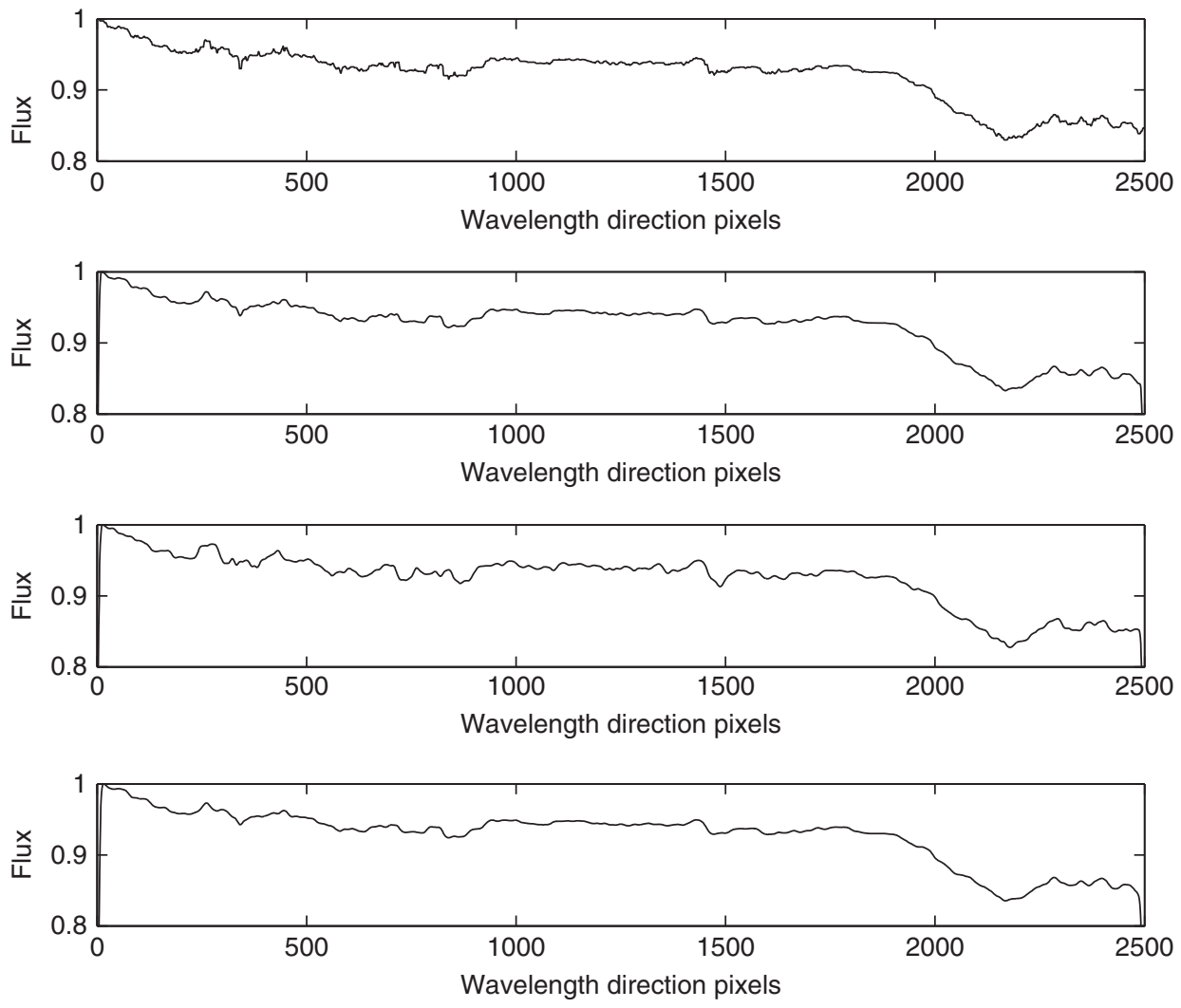

Figure 2 Flux extraction by the spatial extraction method and our method, based on simulated data without noise. From top to bottom, the three sub-figures are in sequence of the standard spectrum, the extracted spectrum in spatial orientation, the extracted spectrum by aperture extraction method and the extracted spectrum by our 2-D model.
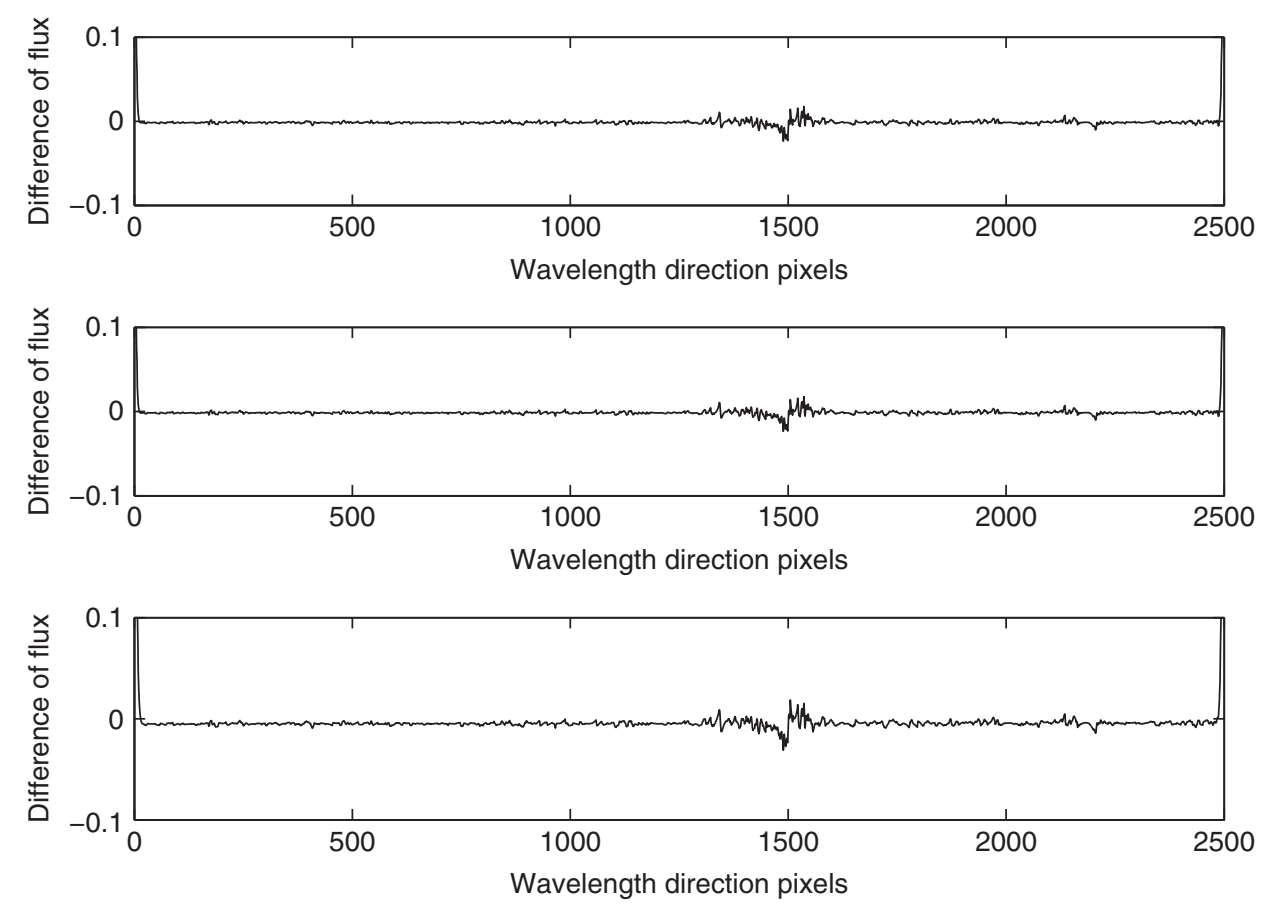

Figure 3 The extracted spectral errors of the three methods. From top to bottom, the three sub-figures are in sequence of the error of the extracted spectrum in spatial orientation, the error of the extracted spectrum by aperture extraction method and the error of the extracted spectrum by our 2-D model. 

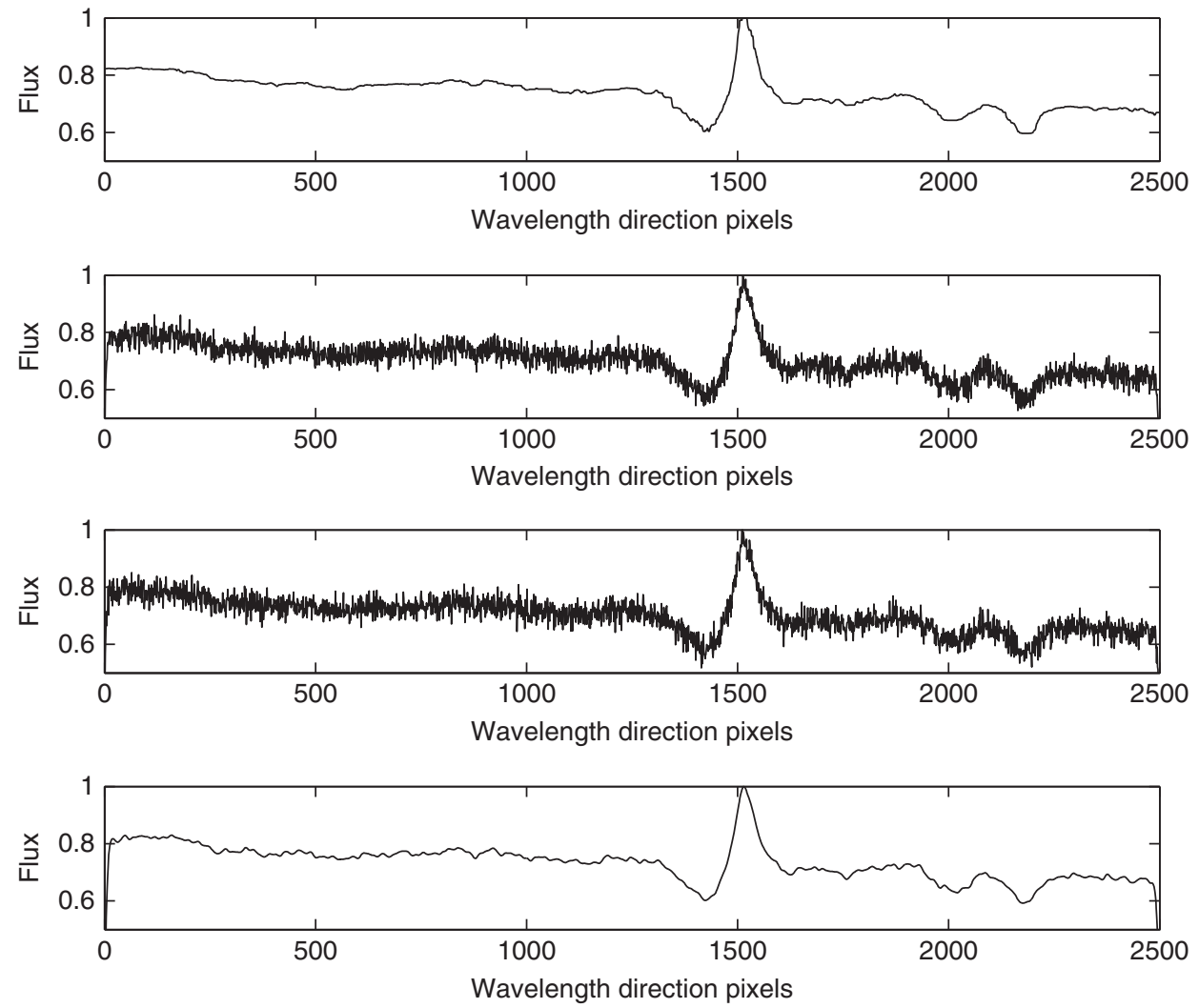

Figure 4 The flux extraction by the spatial extraction method and our method based on the simulated data with noise. From top to bottom the three sub-figures are in sequence of the original spectrum, the extracted spectrum in spatial orientation, the extracted spectrum by aperture method and the extracted spectrum by our 2-D model.

Table 2. The SNR of three spectrum extraction methods with 8-pixel FWHM

\begin{tabular}{lccc}
\hline $\mathrm{SNR}_{\text {in }}$ & 2D model & Gaussian & Aperture \\
\hline 3.03 & 34.09 & 8.99 & 8.36 \\
5.03 & 57.14 & 17.85 & 16.60 \\
8.65 & 104.08 & 35.01 & 34.07 \\
12.04 & 178.05 & 141.56 & 134.2 \\
\hline
\end{tabular}

$$
\mathrm{SNR}_{\text {in }}=\frac{\sum \text { original 2D fiber }}{\sum \mid \text { original 2D fiber }-2 \mathrm{D} \text { fiber with noise } \mid}
$$

More experimental results are given in Table 2. To avoid the effects of different flux base values for different extraction methods, normalization is used. To demonstrate the effectiveness of the spectrum-extraction methods objectively, we define SNR to evaluate the accuracy of the aforementioned several flux extraction methods. The SNR of the spectrum extraction can be expressed as

$$
\mathrm{SNR}=\frac{\sum \text { original spectrum }}{\sum \mid \text { original spectrum }- \text { extracted spectrum } \mid}
$$

The SNR is figured out by the following steps. First, the means of the extracted spectra by the three methods are normalized to eliminate the effect of the relative flux
Table 3. The SNR of three spectrum extraction methods with 4-pixel FWHM

\begin{tabular}{lcrr}
\hline $\mathrm{SNR}_{\text {in }}$ & 2D model & Gaussian & Aperture \\
\hline 1.7268 & 14.51 & 7.10 & 5.76 \\
3.4536 & 27.79 & 13.18 & 10.52 \\
6.9072 & 53.66 & 25.22 & 20.00 \\
34.536 & 211.37 & 113.81 & 93.52 \\
\hline
\end{tabular}

differences resulting from the different methods. Second, the SNR is calculated by Equation 20 for the normalized original spectrum and the extracted spectrum.

Table 3 shows the experimental results of the simulated data with 4-pixel FWHM in the spatial and wavelength directions.

\subsection{Test of Tolerance}

The FWHM of the 2-D normalized model in the spatial and wavelength directions is calculated by the method of Section 2.1. Due to the nonlinear nature of dispersion, the distortion and estimated error, the FWHM may be different at different wavelengths either in spatial direction or in wavelength direction. In this part, the tolerance of our method for the estimated error of FWHM is tested.

First, the tolerance of the estimated error of FWHM in the spatial direction is tested. In this part, the FWHM of the spatial direction at each wavelength pixel takes a 
Table 4. The SNR of three spectrum-extraction methods for tolerance in the spatial direction

\begin{tabular}{lcccc}
\hline$\Delta_{\text {spa }}$ & $\mathrm{SNR}_{\text {in }}$ & 2D model & Gaussian & Aperture \\
\hline 0.5 & 7.98 & 91.37 & 34.20 & 32.21 \\
1.0 & 7.85 & 77.55 & 35.00 & 33.56 \\
1.5 & 7.89 & 53.74 & 30.80 & 32.47 \\
2.0 & 8.01 & 41.33 & 16.94 & 17.23 \\
\hline
\end{tabular}

Table 5. The SNR of three spectrum extraction methods for the tolerance in wavelength direction

\begin{tabular}{lcrcc}
\hline$\Delta_{\text {wav }}$ & SNR $_{\text {in }}$ & 2D model & Gaussian & Aperture \\
\hline 0.5 & 8.03 & 104.70 & 34.83 & 32.85 \\
1.0 & 8.00 & 95.90 & 32.83 & 31.15 \\
1.5 & 7.98 & 82.11 & 30.56 & 29.91 \\
2.0 & 7.01 & 78.39 & 28.01 & 27.79 \\
\hline
\end{tabular}

Table 6. The estimated error test in the spatial direction with 4-pixel FWHM

\begin{tabular}{lcccc}
\hline$\Delta_{\text {spa }}$ & SNR $_{\text {in }}$ & 2D model & Gaussian & Aperture \\
\hline 0.5 & 3.4563 & 26.48 & 12.92 & 10.00 \\
1.0 & 3.4563 & 25.30 & 12.71 & 10.00 \\
1.5 & 3.4563 & 24.25 & 12.47 & 10.00 \\
2.0 & 3.4536 & 23.30 & 12.23 & 10.00 \\
\hline
\end{tabular}

Table 7. The estimated error test in wavelength direction with 4-pixel FWHM

\begin{tabular}{lcccc}
\hline$\Delta_{\text {wav }}$ & SNR $_{\text {in }}$ & 2D model & Gaussian & Aperture \\
\hline 0.5 & 3.4563 & 27.88 & 13.18 & 10.00 \\
1.0 & 3.4563 & 27.84 & 13.19 & 10.01 \\
1.5 & 3.4563 & 27.87 & 13.19 & 10.01 \\
2.0 & 3.4563 & 27.90 & 13.20 & 10.01 \\
\hline
\end{tabular}

random value between $8-\Delta_{\text {spa }}$ and $8+\Delta_{\text {spa }}\left(\Delta_{\text {spa }}\right.$ is a positive value).

Experimental results are given in Table 4 by SNR.

Then, the tolerance of the estimated error of FWHM in wavelength direction is tested. In this part, the FWHM of spatial direction at each wavelength pixel takes a random value between $10-\Delta_{\text {wav }}$ and $10+\Delta_{\text {wav }}\left(\Delta_{\text {wav }}\right.$ is a positive value).

Experimental results are given in Table 5 by SNR.

Tables 6 and 7 are the experiments for the tolerance test of the estimated errors of FWHM in the spatial and wavelength directions, respectively. The PSF of the simulated data has a 4-pixel FWHM in the two directions.

The tolerance for the unsymmetrical fiber profiles of spatial direction will be tested, and the results are given in Table 8. The 2-D PSF which is used to simulated the fiber profiles with the FWHM of spatial direction taking a constant value, $2+\Delta$ and 2 at the center point,
Table 8. The SNR of three spectrum extraction methods for tolerance of radial distortion

\begin{tabular}{lccccr}
\hline$\theta$ & $\Delta$ & SNR $_{\text {in }}$ & 2D model & Gaussian & Aperture \\
\hline 0 & 0.5 & 3.4536 & 26.21 & 12.47 & 10.00 \\
0 & 1.0 & 3.4536 & 24.79 & 11.83 & 10.00 \\
0 & 1.5 & 3.4536 & 23.52 & 11.26 & 10.00 \\
0 & 2.0 & 3.4536 & 22.37 & 10.75 & 10.00 \\
25 & 0.5 & 3.4536 & 27.46 & 13.25 & 9.99 \\
25 & 1.0 & 3.4536 & 27.56 & 13.50 & 9.98 \\
25 & 1.5 & 3.4536 & 27.63 & 13.73 & 9.96 \\
25 & 2.0 & 3.4536 & 27.65 & 13.93 & 9.94 \\
45 & 0.5 & 3.4536 & 28.03 & 13.54 & 9.99 \\
45 & 1.0 & 3.4536 & 27.88 & 13.81 & 9.97 \\
45 & 1.5 & 3.4536 & 27.66 & 14.01 & 9.96 \\
45 & 2.0 & 3.4536 & 27.38 & 14.17 & 9.95 \\
75 & 0.5 & 3.4536 & 26.59 & 13.30 & 10.00 \\
75 & 1.0 & 3.4536 & 26.22 & 13.53 & 9.99 \\
75 & 1.5 & 3.4536 & 25.81 & 13.67 & 9.98 \\
75 & 2.0 & 3.4536 & 25.37 & 13.72 & 9.98 \\
90 & 0.5 & 3.4536 & 27.71 & 13.17 & 9.98 \\
90 & 1.0 & 3.4536 & 27.64 & 13.16 & 9.97 \\
90 & 1.5 & 3.4536 & 27.57 & 13.15 & 9.95 \\
90 & 2.0 & 3.4536 & 27.51 & 13.13 & 9.94 \\
\hline
\end{tabular}

respectively. $\theta$ denotes the degree to the dispersion axis of the radial distortion. $0^{\circ}$ means the radial distortion in spatial direction, and $90^{\circ}$ means the radial distortion in wavelength direction.

\subsection{Discussion for Cross-talk}

For the fiber whose cross-talk affected by adjacent fibers cannot be ignored, the spectrum extraction method using our 2-D Gaussian model is useful.

The parameters of the 2-D Gaussian model can be obtained from flat and calibration lamp images by the method introduce in Section 2.1 by selecting the sampling points close enough to the fiber center to decrease the cross-talk. For the filed images, if the cross-talk cannot be ignored, the parameters of the 2-D Gaussian model in the spatial direction could be calculated by the method based on the radial basic function algorithm (Qin, 2008). This algorithm uses the 1-D Gaussian function of the 2-D model in the spatial direction as the transmitted function, by modifying the sigma of the function to obtain the best parameters of the function. As this algorithm can use multiple 1-D PSFs in the spatial direction of filed images at the same time, the cross-talk of adjacent fibers can be defeated well. For the center fiber of the observed object fibers, which is polluted by cross-talk heavily, we consider its cross-talk only from two adjacent fibers. The spectrum extracting method for the center fiber will be described, and spectrum extraction of other fibers is same.

The sampling points are adopted in the area of the center fiber profile, and the number of the sampling points is $N . M$ denotes the pixels of each fiber along the wavelength orientation. $G_{j i}^{k}(j=1, \ldots, M ; i=1, \ldots, N$; $k=1,2,3)$ denotes the 2-D Gaussian model of the $j$ th center of the $k$ th fiber along the wavelength orientation of 
the fiber projecting onto the $i$ th sampling point. $x_{j}^{k}$ $(j=1, \ldots, M)$ denotes the original value of the $j$ th center along the wavelength orientation of the $k$ th fiber before diffusing in the form of the 2-D PSF, and $x_{j}^{2}$ is the flux extracted from the center fiber. The value of $N$ must be larger than $3 M$ to use the least-squares theory.

$$
\mathrm{G}_{1} \mathrm{X}_{1}=\mathrm{Y}_{1} \text {, }
$$

where

$$
\begin{aligned}
& \mathrm{G}_{1}=\left[\begin{array}{lll}
\mathrm{A}_{11} & \mathrm{~A}_{21} & \mathrm{~A}_{31} \\
\mathrm{~A}_{12} & \mathrm{~A}_{22} & \mathrm{~A}_{32} \\
\vdots & \vdots & \vdots \\
\mathrm{A}_{1 N} & \mathrm{~A}_{2 N} & \mathrm{~A}_{3 N}
\end{array}\right] \\
& \mathrm{A}_{\mathrm{ij}}=\left[\begin{array}{lll}
\mathrm{G}_{1 \mathrm{j}}^{\mathrm{i}} / \sigma_{\mathrm{j}} & \cdots & \mathrm{G}_{M \mathrm{j}}^{\mathrm{i}} / \sigma_{\mathrm{j}}
\end{array}\right] \\
& \mathrm{X}_{1}=\left[\begin{array}{lllllllll}
x_{1}^{1} & \cdots & x_{M}^{1} & x_{1}^{2} & \cdots & x_{M}^{2} & x_{1}^{3} & \cdots & x_{M}^{3}
\end{array}\right]^{T} \\
& \mathrm{Y}_{1}=\left[\begin{array}{llll}
y_{1} / \sigma_{1} & y_{2} / \sigma_{2} & \cdots & y_{N} / \sigma_{N}
\end{array}\right]^{T} \text {. }
\end{aligned}
$$

As the formulae of Equation 17 are over-determined, we can use the method introduced in Section 2.2 to get the center fiber flux.

\section{Conclusion}

In this article, a novel spectrum extraction method is proposed. The model parameters in the spatial and wavelength orientation are obtained by the flat and calibration lamp images, respectively, to compose the 2-D model. Then the flux can be extracted based on the 2-D model and least-squares theory. Through the experiments, the extracting spectra based on the 2-D model have better SNR than those obtained through the single-Gaussian method and the aperture-extraction method, and the new method has strong tolerance for the estimated error of FWHM in spatial and wavelength directions.

\section{Acknowledgments}

This work was funded by the National Natural Science Foundation of China (NSFC grant 11078016). The authors acknowledge support from the Graduate Innovation Fund of USTC KD2008060.

\section{References}

Becker, T., 2001, PhD Thesis, Potsdam University

Bolton, A. S. \& Schlegel, D. J., 2010, PASP, 122, 248

de Boer, K. S. \& Snijders, M. A. J., 1981, IUE NASA Newsletter, 14,154

Deng, T. B., 1997, ICICS, 1288

Horne Keith, 1986, PASP, 609, 617

Marsh, T. R., 1989, PASP, 98, 609

Qin, H. Q., Ye, Z. F. \& Luo A-li, 2009, PASP, 121, 408

Qin, H. Q., Ye, Z. F., Luo A-li, \& Zhang, H. T., Proc. SPIE, Vol. 7019, 701934, 2008

Robertson, J. G., 1986, PASP, 1220, 1231

Roth, M. M., Kelz, A. \& Fechner, T. et al., 2005, PASP, 117, 620

Sanchez, S. F., 2006, AN, 327, 850

Sharp, R. \& Birchall, M. N., 2010, PASA, 27, 91

Zhang, H. T., Chu, Y. Q., \& Chen, J. S. 2001, AcASn, 42, 313 